\title{
Belgeo
}

Revue belge de géographie

1 | 2013

Modelling and benchmarking of borders

\section{Typologie des espaces-frontières à l'heure de la globalisation}

A typology of border areas in the age of globalization

\section{Remigio Ratti et Martin Schuler}

\section{OpenEdition}

\section{Journals}

Édition électronique

URL : http://journals.openedition.org/belgeo/10546

DOI : 10.4000/belgeo.10546

ISSN : 2294-9135

Éditeur :

National Committee of Geography of Belgium, Société Royale Belge de Géographie

Référence électronique

Remigio Ratti et Martin Schuler, «Typologie des espaces-frontières à l'heure de la globalisation », Belgeo [En ligne], 1 | 2013, mis en ligne le 31 octobre 2013, consulté le 30 avril 2019. URL : http:// journals.openedition.org/belgeo/10546; DOI : 10.4000/belgeo.10546

Ce document a été généré automatiquement le 30 avril 2019

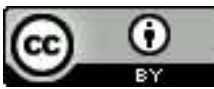

Belgeo est mis à disposition selon les termes de la licence Creative Commons Attribution 4.0 International. 


\title{
Typologie des espaces-frontières à l'heure de la globalisation
}

\author{
A typology of border areas in the age of globalization
}

Remigio Ratti et Martin Schuler

\section{Introduction}

1 L'analyse des régions frontières - et en particulier des espaces-frontières et des politiques transfrontalières - présente des situations très différentes d'un cas à l'autre. Les processus d'intégration politique et la globalisation de l'économie vont dans la direction du redéploiement des vieilles frontières (Schuler et al., 1999), voire de leur effacement, mais cette tendance implique soit des décalages, soit de véritables retours en arrière ou, encore, l'avènement de nouvelles frontières (World Development Report, 2009). La réalité est toutefois si complexe que l'on rencontre des situations apparemment inextricables sans forcément s'interroger sur la nature changeante du rôle des espaces-frontières. La frontière est une construction historique évolutive et un objet spatial en mutation (Espace Temps, 2004) : "La 'frontière' est habituellement comprise comme la "limite de souveraineté et de compétence territoriale d'un Etat”. De nos jours, la prégnance de cette définition semble s'estomper à l'échelle mondiale, accompagnant ainsi le processus de relativisation multiforme de l'Etat. Il faut y voir l'effet de l'évolution des techniques de transport et de communication, la dynamique et l'ampleur des échanges économiques, mais aussi la prise en considération politique d'une plus grande interdépendance du système-monde".

2 Ainsi, en proposant une vision synthétique sous forme de typologie des différentes combinaisons entre visions spatio-temporelles (fixe, mobile, horizon) et approches du pouvoir politique en la matière (ligne; zone; globaliste; cf. figure 1), nous croyons pouvoir offrir une contribution à la théorisation des espaces-frontières.

3 La matrice contient une partie (les quatre premiers cadrans) que l'on peut qualifier de classique parce qu'elle utilise les conceptions courantes de la frontière (Guichonnet et 
Raffestin, 1974), complétée d'une deuxième partie (5 cadrans) qui est le fruit de l'introduction de deux paradigmes nouveaux: celui d'un horizon spatio-temporel complètement ouvert et celui d'une politique "globaliste" du pouvoir politique. En particulier, on constatera toute une série d'effets à caractère fonctionnel qui effacent les effets territoriaux traditionnels ou s'y ajoutent.

\section{La construction d'une typologie des frontières : une combinaison entre conceptions spatiales des espaces du pouvoir politique et des dimensions spatio- temporelles de la frontière}

\section{Considérations introductives}

4 L'article, qui se concentre sur l'élaboration d'une typologie, ne contient que les références spécifiques et renvoie pour la théorie à la littérature générale et aux autres contributions dans ce numéro.

Cette contribution à la théorisation par une typologie représente un pas supplémentaire par rapport à des publications antérieures (Ratti, 1991, 1994, pp. 15-33) en formalisant les combinaisons classiques (Ratti, 2003, pp. 11-14) entre frontières fixes ou mobiles versus frontières lignes de séparation ou zones de contact, en d'autres termes, une typologie valable pour les espaces continus, bien que mouvants et dynamiques.

L'observation des phénomènes en cours concernant la frontière montre une variété d'implications spatiales, en bonne partie mises en évidence aujourd'hui par les processus de globalisation, mais en principe préexistantes. "L'existence de la frontière - affirme l'économiste Claude Courlet (1986) - ne s'apparente pas à un acte arbitraire, mais répond à une logique de projet (de l'économie capitaliste, ndr) qui se joue aussi bien à l'intérieur de l'espace considéré que face à d'autres espaces".

7 Toute une série de facteurs introduisent de nouvelles limites dans la transition entre espaces ruraux et espaces urbains (Leimgruber, 2004). La société moderne, entre global et local, enregistre de profondes modifications dans les dynamiques des périphéries et des zones frontières. La mondialisation a pour conséquence - écrit Denis Retaillé (2012) - de conduire à une présence du Monde partout, ce qui lui permet de considérer trois types de limites : la frontière, le front et l'horizon, selon que ces limites aient deux bords, un bord, ou pas de bord du tout. Gabriel Popescu (2012) arrive à des conclusions analogues: "Borders acquire territorial mobility by being embedded into flows so that bordering can be performed anyplace in the Earth". En quelque sorte, les frontières des flux s'insèrent et agissent à l'intérieur même des territoires, tandis que les zones frontières jouent, dans la globalisation de ce début du $21^{\mathrm{e}}$ siècle, un rôle de transition et de pont, voire dans certains cas de catalyseur, dans la connexion entre États.

Dans ce contexte actuel de bouleversements, l'analyse des frontières exige de mettre l'accent sur les processus et sur les phénomènes de mobilité (Popescu, 2012) exprimés par une demande simultanée, paradoxale, de liberté de mouvement et de sécurité territoriale rassurante. Il devient ainsi de plus en plus évident de considérer les traces laissées dans l'horizon ouvert des flux et des réseaux de la globalisation et de saisir les modifications dans la géographie des espaces-frontières, sans perdre de vue que le résultat sera celui 
d'une grande variété de frontières. Ceci pousse à persévérer dans le choix d'une construction typologique.

9 La prise en considération dans notre nouvelle lecture des concepts d'"horizon" et de dimension "globaliste" élargit le cadre en produisant neuf cadrans types au lieu des quatre de l'approche traditionnelle. A partir des frontières continues de l'approche classique, l'analyse vient ajouter les nouvelles frontières des espaces fonctionnels engendrés par les relations économiques, sociales et politiques désormais insérées dans la globalité.

\section{La construction d'un schéma typologique postmoderne des frontières}

10 Notre schéma typologique postmoderne des frontières combine des espaces contigus de l'approche classique avec des espaces à horizon ouvert de l'approche globaliste.

Figure 1. Typologie des frontières selon leurs conceptions politiques du pouvoir et leur nature spatio-temporelle.

\begin{tabular}{|c|c|c|c|c|}
\hline & & \multicolumn{3}{|c|}{ Dimensions spatiales des limites du pouvoir politique } \\
\hline & & LIGNE & ZONE & GLOBALISTE \\
\hline 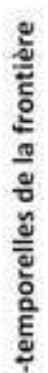 & MOBILE & $\begin{array}{l}\text { Les frontières des } \\
\text { (2) } \\
\text { Régimes (néo) } \\
\text { colonialistes }\end{array}$ & $\begin{array}{l}\text { Les frontières des } \\
\qquad \begin{array}{l}\text { (4) } \\
\text { Politiques de } \\
\text { Régionalisation }\end{array}\end{array}$ & $\begin{array}{c}\text { Les frontières des } \\
\text { (7) } \\
\text { Sites et } \\
\text { Localisations non- } \\
\text { banales } \\
\text { Les frontières des } \\
\text { (8) } \\
\text { Lieux et des } \\
\text { Réseaux }\end{array}$ \\
\hline 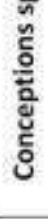 & $\begin{array}{l}\text { OUVERTE } \\
\text { a } \\
\text { L'HORIZON } \\
\text { (anytime/ } \\
\text { anywhere) }\end{array}$ & $\begin{array}{l}\text { Les frontières des } \\
\text { (5) } \\
\text { Pratiques } \\
\text { discriminatoires }\end{array}$ & $\begin{array}{l}\text { Les frontières des } \\
\quad(6) \\
\text { Aires d'influence }\end{array}$ & $\begin{array}{l}\text { Les frontières des } \\
\text { (9) } \\
\text { Places et des } \\
\text { Cités } \\
\text { O hatt schuler } 2013\end{array}$ \\
\hline
\end{tabular}

11 La lecture spatiale des espaces-frontières doit être vue dans un contexte de "champ de forces” de nature multiple : politique, économique, sociale. En général, c'est le pouvoir politico-institutionnel qui aspire à encadrer ces champs de forces en faisant valoir - de façon formelle ou informelle - ses règles de jeu. Nous privilégions donc la dimension politique, vue dans ses implications territoriales et spatiales, pour en faire un axe de notre matrice typologique des frontières.

12 Sur ce premier axe horizontal de la matrice, nous plaçons d'abord les deux approches classiques (et non nécessairement exclusives) qui définissent les modalités et les limites $d u$ pouvoir politique en matière de frontière.

13 D'abord, la frontière ligne: cette frontière linéaire affirme un principe de séparation, en particulier comme ligne de démarcation entre systèmes politico-institutionnels différents. Ce concept est essentiellement le fruit de préoccupations d'ordre national et 
de politiques internationales qui relèvent du pouvoir central de l'Etat national, avec des conséquences pénalisantes pour le "pouvoir régional" qui devrait intervenir dans la gestion de ses relations externes. L'effet frontière se manifeste dans les trois fonctions classiques suivantes (Guichonnet et Raffestin, 1974) : la fonction légale, dont la ligne de frontière délimite exactement les territoires assujettis aux normes juridiques et aux règles de l'Etat; la fonction de contrôle, qui caractérise tout passage de la frontière ; la fonction fiscale, qui assure par la perception de droits (de douane, impôt sur la valeur ajoutée, autres taxes) l'adaptation au droit fiscal en vigueur.

Ensuite, la frontière peut être vue en tant que vecteur de contact et, dans ce sens, elle ne s'exprime plus en tant que ligne, mais est bien un espace, une zone, fonctionnant comme élément de couture (Courlet, 1988, pp.5-12) et d'intermédiation entre sociétés et collectivités différentes. Dans ce cas, on peut parler de région transfrontalière parce que les préoccupations visent l'organisation de l'espace et sa gestion globale, tout en tenant compte des facteurs socioculturels et identitaires.

Non sans raison, ces approches remontent toutes aux années 70/80 du siècle dernier, avant la globalisation et la montée de l'importance des flux et des réseaux, ce qui laisse entendre une modification de la nature des frontières. Nous introduisons alors une nouvelle approche que nous appelons globaliste, selon les définitions d'Ulrich Beck (1997). Insistons sur le fait qu'une approche "globaliste" ne fait pas référence au "globalisme", qui correspond à l'idéologie néolibérale et à la primauté des marchés, tandis que l'approche globaliste renvoie au concept de "globalité", par lequel nous entendons la conscience de vivre dans une société mondiale tout en vivant en même temps les différences et les multiplicités qui nous caractérisent.

Sur l'axe vertical de la matrice, nous représentons les différentes conceptions spatiotemporelles à prendre en considération.

D'abord, considérons les conceptions classiques de frontière fixe ou de frontière mobile. Si dans notre arrière-pensée westphalienne la frontière d'un Etat est en principe bien fixe, l'histoire nous montre au contraire bien de situations de fluidité, que ce soit dans un contexte géopolitique de conquête spatiale (de nature militaire, politique ou économique) ou bien dans celui du front pour lequel l'historien américain Frederick Turner $(1893,1921)$ a jugé normal que la construction des Etats-Unis d'Amérique ait été une histoire de progression de l'Est vers l'Ouest.

18 Ici aussi, nous ne restons pas ancrés aux visions classiques. Nous nous appuyons sur des réflexions récentes sur la mondialisation et les frontières, notamment la considération des trois types de limites : la frontière, le front et l'horizon, selon que ces limites aient deux bords, un bord, ou pas de bord du tout (Retaillé, 2011, 2012). Il nous a semblé tout à fait conséquent d'ajouter pour la construction de notre schéma typologique cette troisième vision spatio-temporelle, en adoptant ce concept d'horizon ouvert.

Le résultat donne une combinaison de situations types - voir figure 1 - selon les différentes visions politiques du pouvoir et leur conception spatio-temporelle, que nous analyserons dans la figure $2 \mathrm{du}$ point de vue de leurs caractéristiques: fonctions, processus de différentiation et gestion éventuelle des discontinuités.

\section{Les traits caractéristiques des différents types de frontières}


Figure 2. Typologie des espaces-frontières et leurs caractéristiques : (1) fonctions ; (2) processus de différentiation ; (3) gestion éventuelle des discontinuités.

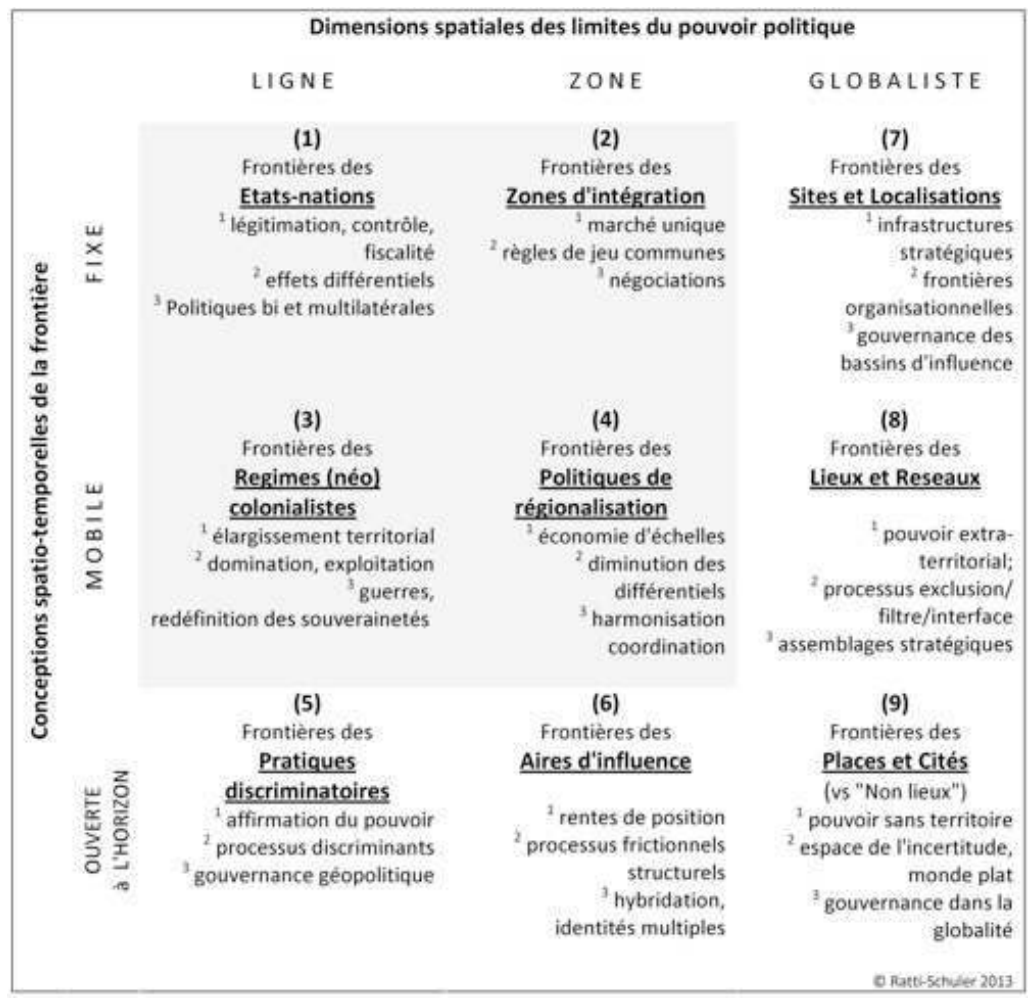
spatio-temporelles fait état de nouvelles frontières de nature fonctionnelle: on s'éloigne des cas classiques relevant de situations représentées par des espaces contigus pour aller vers une nouvelle perception des territorialités et vers des souverainetés fragmentées par de nouveaux espaces-frontières.

Si dans les cadrans précédents $\mathrm{N}^{\circ} 5$ et 6 , l'espace apparaît encore dans sa dimension institutionnelle traditionnelle de ligne de démarcation et de zone-espace de souveraineté, il perd ses traits de continuité face à la dimension fluide de l'approche globalise (cadrans des types 7, 8 et 9). En suivant Castells (2006), l'espace postmoderne tend bien à effacer ou à bouleverser les vieilles territorialités, mais il ne devient toutefois pas, selon les termes de Marc Augé (1992) un "non lieu". Si, d'une façon sectorielle et fonctionnelle, l'espace voit apparaître de nouvelles formes d'appropriation en termes de sites, de localisations, de lieux et de réseaux qui, pour finir (cadran 9), sont encadrés par des places et des cités à l'échelle métropolitaine des processus de globalisation, à nouveau, dans ces cas de figures, se dessine l'émergence de frontières de natures nouvelles qui tendent à dépasser celles $\mathrm{du}$ pouvoir des Etats-nations pour s'insérer dans le scénario fonctionnel d'une gouvernance locale-globale.

\section{La typologie classique des frontières considérées dans un espace continu}

Tout en prenant en considération les définitions de frontières devenues classiques et que nous donnons pour acquises, nous pouvons imaginer d'abord une construction qui 
combine sur les deux axes cartésiens les différentes fonctions de la frontière - "ligne de séparation" et "zone de contact" - avec ses applications spatio-temporelles "fixes" et "mobiles". Il en résulte une première combinaison en quatre cadrans (de 1 à 4 dans les figures) des types classiques de frontières, ayant pour caractéristique de se situer dans un espace continu.

23 1. Le premier cadran - "Frontières des Etats-nationaux" - relève du domaine primordial des frontières politico-institutionnelles des Etats nationaux westphaliens ${ }^{1}$. Dans ce contexte, la frontière est à la fois fixe et ligne de séparation entre des systèmes politiques souverains ; elle est accompagnée d'une fonction de contrôle et d'une fonction douanière et fiscale. Ce type de frontière correspond à la phase historique de la construction des Etats-Nations et aux modèles traditionnels de la théorie des échanges "inter-nationaux", entre entreprises et nations relevant de systèmes politiques et économiques différents.

La réalité de la frontière des Etats-nations à donné lieu à des modèles différents, en fonction de la combinaison d'intérêts internes et de leur juxtaposition avec les intérêts de l'Etat ou des Etats limitrophes :

24 - l'intérêt militaire est d'avoir des frontières faciles à défendre (logique du pouvoir et de la stabilité);

- l'intérêt de l'économie est celui du profit, correspondant aujourd'hui (loin du mercantilisme historique et contre l'option protectionniste) plutôt à la politique libérale des frontières facilitant l'échange (logique de la spécialisation et de la compétitivité internationale).

Ainsi, la France semble bien avoir utilisé l'idéal de la "géographie des frontières naturelles" pour construire les frontières, tandis que celles de la Suisse dépassent les Alpes et coupent les aires urbaines (Genève, Bâle). D'autres "nations" traversant les massifs ont disparu ou ne se sont jamais créées (Piémont-Sardaigne, Kurdistan).

Dans le contexte typologique "Etat-nation", toute collaboration transfrontalière a un caractère interétatique. Les relations des zones transfrontalières sont dépendantes, en principe, des pouvoirs des autorités politiques au niveau national. Ainsi, les instances de la collaboration transfrontalière qui se sont développées au cours des dernières décennies en Europe - démarrées avec la Convention de Madrid du Conseil de l'Europe de 1980 - ont impliqué une adhésion et une ratification des Etats.

2. Le deuxième cadran - "Frontières des zones d'intégration" - combine la volonté politique de construire de nouveaux espaces élargis d'action institutionnelle - dont le caractère supranational peut avoir différentes formes (Union; Confédération d'Etats ; constitution fédérale régionale) - tout en maintenant des frontières fixes, distinguant nettement l'appartenance ou l'exclusion. Le processus d'intégration européenne du deuxième aprèsguerre du siècle dernier nous présente l'exemple de déclinaisons progressives du principe d'intégration à la recherche de règles de jeu communes: de l'union douanière à la création du marché unique, en passant par les processus visant l'Union économique et monétaire.

Le dépassement des discontinuités provoquées par les espaces-frontières relève du domaine de négociations bilatérales ou multilatérales. C'est le cas de l'Espace Economique Européen (EEE), fruit d'un accord signé en 1992 et entré en vigueur en 1994, entre la Communauté européenne de l'époque et les pays de l'Association européenne de libreéchange. Cet accord est conçu (Degryse, 2007) pour permettre aux pays voisins de la Communauté de bénéficier des avantages du marché intérieur européen. En contrepartie, les pays de l'AELE qui participent à l'EEE (la Suisse a dû y renoncer suite au vote 
référendaire négatif du peuple suisse le 6.12.1992) doivent accepter les règles du marché unique. La Suisse s'est vue contrainte à plusieurs phases d'importantes négociations bilatérales, dont la poursuite et l'extension pourraient se trouver remises en question. Mentionnons encore que la Suisse, bien que pays tiers, participe à l'espace Schengen/ Dublin impliquant la suppression des contrôles aux frontières intérieures et réglant les questions de l'asile et de l'immigration.

3. Le troisième cadran - "Frontières des régimes (néo)colonialistes" - nous introduit, en combinant la frontière conçue comme ligne de séparation avec une frontière qui se veut mobile, dans un contexte géopolitique de conquête spatiale, que ce soit de nature militaire, politique ou économique. Relèvent de la même logique les processus de colonisation du $19^{\mathrm{e}}$ au milieu du $20^{\mathrm{e}}$ siècle, ainsi que les phénomènes d'alliances visant la création d'espaces d'hégémonie et de pouvoir militaire, politique ou économique.

Les processus de colonisation sont complexes et des intérêts différents s'y jouent (Agnew, 2009, pp. 109-110). Ils peuvent s'exprimer par les drapeaux hissés dans des territoires inoccupés ou sous-occupés, ainsi que par l'imposition des souverainetés à des nomades qui avaient le seul tort de ne pas revendiquer une terre qui était bien la leur, et finalement par les lignes de partage de souveraineté entre Etats dominants. La fixation de frontières de la colonisation et de la décolonisation relève de deux formes :

- des tracés linéaires avec peu de considération des intérêts (et de leur conception spatiale) de la population indigène ${ }^{2}$ qui deviennent des frontières linguistiques, puis des frontières nationales après l'Indépendance (en Amérique du Sud dès 1810, en Afrique à partir de 1960) ;

- des tracés "savants", basés sur des recensements ou des travaux ethnographiques (Asie centrale de l'URSS) qui deviennent des frontières nationales (1991).

La résolution des crises provoquées en termes de domination et d'exploitation par les régimes de colonisation se traduit souvent en conflits armés à l'intérieur et à l'extérieur des aires concernées tandis que la redéfinition des frontières et des souverainetés subit les hypothèques de la hiérarchie des puissances dominantes.

4. Enfin, le quatrième cadran - "Frontières des espaces de régionalisation" - ressort de la combinaison d'une frontière perçue comme un espace de contact et à géométrie variable. C'est une zone en mouvance à la recherche d'une meilleure cohérence systémique (économies d'échelle notamment), combinant un pluralisme des structures et des identités. Cela présuppose une modification ou une adéquation régionale des fonctions encore attribuées aux frontières de l'Etat national. Comme souligné par W. Leimgruber (2004), la société moderne change la vision de marginalisation des régions frontière, en combinant forces centripètes et centrifuges, logiques politiques et logiques économiques. Dans l'exemple des régions de coopération transfrontalière (au sens du Conseil de l'Europe), ou, en particulier, entre l'UE et pays tiers (programmes INTERREG), on reste en présence d'une ligne fixe de démarcation des frontières institutionnelles tout en poursuivant une politique de correction des "cicatrices de l'histoire" - selon les termes de Denis de Rougemont (1971, pp.9-12) - voire de coopération et d'intégration régionale transfrontalière. On passe d'une économie de zone frontière juxtaposée à une économie "transfrontalière" qui présuppose une coopération entre deux systèmes nationaux et la résolution de nombreuses tensions ressortant souvent d'importants effets différentiels : différences dans les niveaux des prix, dans les revenus et taux salariaux, dans la protection sociale et des services à la population ou, encore, dans la dotation infrastructurelle. Selon la terminologie bien établie de L. Senn (1990), il s'agit de dépasser 
les coûts de la non-coopération: (i) coûts de duplication (infrastructure parallèles juxtaposées); (ii) coûts de non réalisation d'économie d'échelle; (iii) coûts d'accords manqués ou retardés (par exemple dans l'épuration des eaux); (iv) coûts de programmation adverse; (v) coûts de droit de veto, notamment des niveaux institutionnels supérieurs.

Le passage à une vision transfrontalière n'est toutefois pas évident : dans un contexte de zone frontière juxtaposée, une des parties est peut être gagnante, l'autre perdante, ceci parfois à tour de rôle selon les règles de jeu des Etats-nations, les phases de développement ou la conjoncture qui peuvent engendrer des rentes de position (de "localisation" par exemple) et/ou des rentes différentielles (par exemple la contrebande, les différentiels salariaux ou fiscaux).

L'économie transfrontalière" tend au contraire à changer d'échelle, plaçant les réalités socioculturelles régionales dans la possibilité de forger de nouvelles identités et des structures économiques à meilleure valeur ajoutée réelle.

\section{La typologie postmoderne des frontières considérées dans une vision à horizon ouvert et dans une approche globaliste}

En continuant la description, pas à pas du tableau typologique en neuf cadrans, cinq autres cas de figures viennent s'ajouter si l'on considère les nouveaux éléments de fluidité - "anytime" et "anywhere", introduits par la révolution technologique et organisationnelle du domaine informatique et de la communication. Dans le monde des flux de la globalisation les frontières traditionnelles des espaces contigus sont mises en question par des processus fort contradictoires qui vont de leur effacement à la construction de nouvelles frontières fonctionnelles - faisant suite à l'apparition de nouveaux champs de force du pouvoir dans un scénario à horizon ouvert.

1. Le cadran $\mathrm{N}^{\circ} 5$ "Frontières issues de pratiques discriminatoires" de notre schéma typologique fait état de forces politiques qui, même dans un scénario à horizon ouvert, veulent exercer de nouvelles lignes fonctionnelles de démarcation en répondant, en particulier, à l'intrusion épidémique de phénomènes de portée spatiale globale. Il s'agit de pratiques instrumentales, souvent non-gouvernementales, qui ont le but d'affirmer un pouvoir - économique, socio-culturel, politique ou militaire (cf. ci-après "Considérations finales sur la suite des recherches") en reconstruisant des territorialités qui en principe seraient effacées par des processus de la globalisation.

Dans le domaine de l'informatique et des technologies de la communication - par définition celles qui sont rendues disponibles, partout et en même temps, par la révolution de la numérisation de l'élaboration, la diffusion et le stockage des données les exemples de pratiques discriminantes sont légion. C'est en général le cas de groupements d'intérêts, soit gouvernementaux, soit non-gouvernementaux, qui introduisent et sont capables d'imposer des régimes et des normes de différentes natures: fixations de protocoles spécifiques de mise en réseau (dans le domaine des communications, par exemple); recherche de standards de qualité (normes ISO), de sécurité, de protection écologique et environnementale.

Dans le domaine des nouveaux médias électroniques, il est facile de trouver des exemples de pratiques de surveillance, voire de censure, imposées par leurs protagonistes pour 
sauvegarder des intérêts commerciaux ou politiques. Le brouillage des chaînes de télévision ou de programmes, ainsi que la volonté de garder un contrôle national dans le domaine de l'information et des droits d'auteurs constituent autant de pratiques qui ne permettent certainement pas de parler - malgré les slogans - de télévision sans frontières.

Les batailles que se livrent les entreprises du secteur des TIC ont une valeur géopolitique qui, dans l'intérêt des usagers, demanderait aussi une gouvernance particulière au niveau global.

31 2. Le cadran-type $\mathrm{N}^{\circ} 6$ "Frontières des aires d'influence" permet de décrire, malgré le contexte d'un horizon ouvert, les frontières qui trouvent leur explication dans l'existence de caractéristiques structurelles des sociétés, voire de stratifications laissées par l'histoire (on pourrait les appeler "plaques tectoniques"), bien identifiables, également en termes de pouvoir politique, dans des aires déterminées quoique perméables.

Historiquement, cette situation est bien présente dans la notion d'économie-monde de Ferdinand Braudel ; en le citant, Courlet (1996) montre que "toute économie-monde a ses limites économiques : celles-ci se situent là où commence une autre économie-monde, le long d'une ligne, mieux d'une zone que, d'un côté comme de l'autre, il n'y a avantages, économiquement parlant, à franchir que dans des cas exceptionnels... $1 \mathrm{l}$ faut vaincre l'espace... et l'économie monde déplace ses limites pour incorporer de nouvelles zones jusqu'ici restées en dehors de son influence".

Tout en étant confronté aux processus d'aplatissement du monde mis en évidence par T. Friedman (2006), l'espace anthropologique reste différencié par les traits structurels qui caractérisent l'histoire des territoires. Ceux-ci déterminent des "espaces zone", caractérisés par des conditions cadre et des normes qui les rendent moins perméables en déterminant des obstacles de nature frictionnelle ou des phénomènes de réflectivité (en analogie avec la physique de la lumière).

Parmi les nombreux exemples, prenons de cas du "digital divide" - du fossé numérique. $\mathrm{Si}$, théoriquement, tout le monde est en mesure de bénéficier au moins des instruments les plus élémentaires (un PC à cent dollars) de la technologie numérique, on constate l'existence de véritables frontières liées à des processus biaisés par des éléments de nature frictionnelle et structurelle. Dans le cas de la diffusion d'internet, les frontières frictionnelles sont déterminées non seulement par l'existence de disparités économiques et de pouvoir d'achat, mais surtout de pré-conditions pénalisantes au niveau de la formation et de la culture.

De façon plus générale, au niveau économique, après des décennies de politiques d'intégration et de libéralisation qui ont rendu stratégiques les quatre libertés de circulation - des personnes, des capitaux, des biens et des services -, on assiste à la persistance ou, de plus en plus, à l'apparition de processus frictionnels empêchant les convergences nationales ou démontrant de graves effets structurels indésirables Des constatations analogues peuvent être dressées dans d'autres approches sociologiques, culturelles et politiques (Bonvecchio, 2013, pp. 37-50). En conséquence, on assiste à l'émergence de frontières fonctionnelles exprimées par des aires d'influence, présentant toutefois des degrés de perméabilité différents et laissant la place à des formes d'hybridation et à des identités multiples.

32 3. Le cadran type $\mathrm{N}^{\circ} 7$ "Frontières des sites et des localisations durables" présente le cas des sites et des localisations non banales (c'est-à-dire qui ne peuvent se retrouver n'importe où) qui servent de support à la mobilité internationale des biens et des 
hommes: les grandes infrastructures nodales (ferroviaires ou routières), les ports qui accueillent les immenses navires des voies mondiales de la communication maritime; les grands aéroports plaques tournantes de la mobilité intra et intercontinentale. Ces "nonlieux", ces "places de transition" (Kemp, 2009) sont l'objet d'une attention croissante quant à leur rôle, pas seulement économique. Ils constituent des infrastructures et surtout des assemblages logistiques clés pour les processus de globalisation et déterminent de nouvelles frontières fonctionnelles à caractère organisationnel.

Chacune de ces grandes infrastructures a son bassin de référence et impacte considérablement le développement régional. Par certains aspects, une analogie historique peut être faite avec l'organisation de la Ligue hanséatique. Les espacesfrontières sont mis en évidence par les barrières à l'entrée et les règles du jeu spécifiques qui gèrent ces ensembles et groupements de la logistique continentale et mondiale. La gestion de ces espaces-frontières exige une véritable gouvernance stratégique, publique et privée, qui dépasse largement les capacités du pouvoir politique des Etats-nations.

4. Le cadran type $\mathrm{N}^{\circ} 8$ "Frontières fonctionnelles de réseaux" bouleverse la théorie traditionnelle des espaces-frontières en considérant les phénomènes de diffusion spatiale en réseau et dans un contexte de frontières institutionnelles mobiles, voire flexibles et/ou perméables.

La littérature, faisant écho aux mutations de la ville contemporaine, a conceptualisé ces phénomènes en termes de "ville en réseau" (Castells, 2008) et fait état d'une dialectique entre réseaux et lieux et d'une vision tendanciellement dominatrice des espaces des flux sur les lieux (Castells, 2008), en tant qu'espaces de vie et de l'expérience. La manifestation d'un pouvoir "extra territorial" se lit - en termes de frontières - dans les nouvelles différentiations et dans la rupture des solidarités de proximité, jusqu'à pouvoir signaler un usage corporatif des lieux (Silveira, 2010). Les lieux "sont au croisement éphémère de mouvements continus qui animent le monde" (Retaillé, 2011). Toutefois la géographie et la sociologie récente montrent la formation et la capacité de créer de nouveaux lieuxespaces de vie, en retrouvant même dans les localisations désormais classées "non-lieux" (shopping centers, gares, espaces multimodaux) "des lieux d'appropriation sociale, des lieux de rassemblement, d'échanges, parfois violents, des lieux d'expression publique". "Face au développement urbain mondial, les lieux de l'expérience ne disparaissent pas, mais se démultiplient à travers des formes urbaines et sociales, de plus en plus variées" (Pflieger et al., 2013).

A l'échelle de la globalité, la nouvelle géographie des frontières se lit d'emblée dans l'approche de Saskia Sassen en termes de nouveaux assemblages T.A.D. - Territoire, Autorité, Droit - définissant une troisième dimension de l'espace contemporain qui caractérise les processus non banaux de globalisation. Selon Sassen (La globalisation centrifuge, 2009), la troisième dimension de l'espace contemporain signifie que les transformations en cours vont au-delà de la configuration binaire global-national. De nouveaux assemblages spécialisés des trois composantes "territoire, autorité, droit (T.A.D.)" font leur apparition dans un monde dominé par les relations fonctionnelles de l'économie.

Ainsi cette troisième dimension de l'espace contemporain n'est ni globale, ni nationale. L'équation traditionnelle - T.A.D. = Etat-Nation - se transforme aujourd'hui en nouveaux "assemblages T.A.D. public-privés", par la construction de réseaux fonctionnels et transnationaux, compétitifs et souvent exclusifs. Les exemples les plus parlants sont représentés par la globalisation de la finance mondiale, avec ses grandes places financières et ses acteurs "too big to fail", mais aussi par le commerce mondial des 
matières premières ou les empires de la communication et des nouveaux médias.

Les nouvelles frontières de la discontinuité engendrés par les réseautages fonctionnels de la globalisation remettent en cause la capacité de construire des ponts, de recomposer les mobilités et les proximités (Castells, 1996). Cela appelle des réponses en termes de nouvelle gouvernance et de nouveaux assemblages régionaux du pouvoir (Allen and Cochrane, 2007).

5. Le Cadran $\mathrm{N}^{\circ} 9$ "Les frontières des Places et des Cités" semble bien donner une réponse au modèle théorique qui voudrait que les frontières s'effacent dans la dimension "anytimeanywhere" du monde numérique et des "non lieux". Thomas L. Friedman dans "The Word is Flat" (2005, pp. 51-199) présente les "dix forces qui aplatissent le monde" : une est de nature géopolitique (la chute du Mur de Berlin); deux de nature technologique (la connectivité et la standardisation des protocoles informatiques); cinq de nature organisationnelle (téléchargement; outsourcing; offshoring; les filières de production; la logistique mondiale); une de nature individuelle (la capacité de construire et d'exploiter un réseau personnalisé d'informations et de connaissances) et enfin la convergence (plateforme globale permettant toute forme de collaboration multiple). Toutefois, Friedman même se distancie de son titre et des dérives de la vulgarisation en écrivant (p. 204): "No, not everyone has access yet to this new platform, this new playing field. No, when I say the world is being flattened, I don't mean we are all becoming equal. What I do mean is that more people in more places now have the power to access the flat-world platform - to connect, compete, collaborate, and, unfortunately, destroy - than ever before".

On retrouve alors les thèses bien débattues ces dernières années sur la formation de nouveaux espaces de proximité et de cohérence. Pour J. Agnew (2009, pp. 35-37) on peut, même à l'heure actuelle, retrouver la "piazza" historique, la place : un territoire qui en tant que résultat de l'interaction humaine (dynamique et en changement continu) devient un milieu capable non seulement de soutenir l'organisation politique et économique en rapport au local (place making), mais aussi d'être un pont médiateur avec d'autres réalités spatiales. Celles-ci peuvent être les cités, les "cities", décrites par S. Sassen (2010) comme des points de connexion d'une réalité géographique plus large, dans de réseaux où les cités sont distantes et souvent hautement spécialisées.

Dans ce contexte globaliste, les frontières ne disparaissent pas mais, dans un horizon ouvert, elles sont représentées par la dimension interstitielle d'un monde spécialisé et compartimenté. Elles sont ainsi l'expression de discontinuités qui semblent bien représenter la règle dans les diversités qui caractérisent la globalité, mais qui demandent toutefois une nouvelle gouvernance à différentes échelles spatiales.

\section{Considérations finales sur la suite des recherches}

Le tableau typologique ici présenté est le produit, tout frais, d'une combinaison de réflexions classiques et nouvelles. Cet essai de synthèse typologique demande une phase d'expérimentation appliquée, qui permettra de vérifier et perfectionner son bien-fondé ${ }^{3}$; en particulier, ce travail doit être fait pour chaque case, où on pourra situer une grande variété de situations et de processus à la lumière de différentes approches théoriques.

A titre d'exemple, et pour stimuler et élargir la réflexion, les auteurs se sont demandé s'il était possible d'utiliser la typologie pour essayer d'analyser et de classer des phénomènes spatiaux liés aux différentes formes, anciennes et nouvelles, de pouvoir militaire et d'exercice de la force. Ainsi, à la logique des armées conventionnelles correspondant au 
concept des Etats-nations (cadran 1), répond la logique de régimes impérialistes avec frontières mobiles (colonisation, cadran 3), dotées de suprastructures politico-militaires (par l'exemple l'OTAN, cadran 2) en définissant les nouvelles limites extérieures d'un pacte.

A l'heure de la globalisation et de révolutions technologiques, l'exercice du pouvoir s'est multiplié et différencié. En gardant la conception spatiale de la ligne, un Etat peut exercer des pratiques discriminatoires (cadran 5) en s'appuyant sur sa force technico-militaire, et désigner ainsi de nouvelles démarcations de son pouvoir. On pourrait être tenté de considérer que des contre-pouvoirs procèdent de la même manière, par exemple des cartels de la drogue.

Sous un aspect de contrôle stratégique, les forces militaires sont de moins en moins ancrées dans l'espace d'une territorialité mais centrées sur les lieux stratégiques, hautement contrôlés et protégés (cadran 7).

La réaction des Américains aux attentats du 11 septembre 2001 est l'expression d'une globalisation de la menace et de l'exercice mondial du pouvoir. A-spatial mais ubiquitaire, le terrorisme (Agnew, 2009, pp. 57-59) est un objet assez controversé du point de vue de la recherche (Crettiez et Mucchielli, 2010).

En particulier, quelles sont les frontières et comment peut-on classer l'activité d'organisations qui s'insèrent dans les territoires politiques reconnus et qui, dans les cas extrêmes, exercent un pouvoir parallèle et difficilement contrôlable sur un territoire? Selon notre typologie de la conception des limites du pouvoir et du contre-pouvoir de la force armée, l'appréciation spatiale des frontières de ces phénomènes pourrait trouver un cadre d'analyse de structures qui seraient propres aux cadrans 6 et 8 et qui amèneraient au cadran 9, celui des pouvoirs dans des territoires et espaces d'incertitude.

Pour terminer, notre exercice typologique fait appel à de nouvelles clés de lecture théoriques, capables de nous éclairer sur la complexité, la fragmentation et les discontinuités des vieux et des nouveaux espaces-frontières.

\section{Conclusions}

Qu'en est-il des espaces-frontières à l'ère de la globalisation, à l'ère de la "société liquide" (Bauman, 2002) et du "anytime" et "anywhere" ?

On assiste à des processus de redéploiement des espaces qui en partie dépassent et/ou simplement oublient les territoires contigus des Etats-Nations, bien qu'ils soient la résultante d'une construction de longue durée. ${ }^{4}$

Les mutations spatiales $\mathrm{du} 21^{\mathrm{e}}$ siècle bouleversent les zones frontières : la globalisation introduit de nouveaux éléments fonctionnels dans la définition des frontières et de leurs effets. On observe l'avènement - à côté des effets, souvent stratifiés et contradictoires, des vieilles frontières - de nouvelles frontières qui, malgré l'évocation d'un monde sans frontières, caractérisent aussi le monde des flux et des réseaux. Mais le changement de nature de ces frontières et de leurs effets est fondamental à cause de toute une série d'effets à caractère fonctionnel : l'économie différencie davantage les espaces ou elle les redéfinit par ses réseaux, tandis que l'histoire laisse ses empreintes à travers des effets structurels et que la politique et la société imprègnent l'espace par leurs discriminations.

Cet exercice combinatoire autour des différentes acceptions des concepts de frontière et de son application spatio-temporelle est une approche qui permet de développer, avec de 
nouveaux paradigmes et par de degrés de liberté élargis, un cadre d'analyse mieux adapté aux scénarios possibles de la complexité des situations actuelles. Ces changements sont encore peu perçus; notre approche typologique n'est qu'un outil pour des réflexions ultérieures visant soit l'appréciation théorique soit le traitement des espaces-frontières.

\section{BIBLIOGRAPHIE}

ALLEN J. and COCHRANE A. (2007), "Beyond the Territorial Fix: Regional Assemblages, Politics and Power", Regional Studies, 41, 9, pp. 1161-1175.

AGNEW J. (2009), Globalization and Sovereignty, Rowman \& Littlefield Publishers, New YorkPlymouth (UK).

BAUMAN Z., (2002), Modernità liquida, Laterza, Roma-Bari.

BECK U. (1997), Was ist Globalisierung? Irrtümer des Globalismus - Antworten auf Globalisierung, Ed. Italiana, Che cos'è la globalizzazione? Carocci, 1999, Roma.

BONVECCHIO C. (2013) in Alberti G. (a cura di), La frontiera scomparsa : globalizzazione e cultura dopo la crisi. "Entgrenzung" : indipendenza, spostamento e scomparsa delle frontiere? Mimesis Edizioni, Milano-Udine.

CASTELLS M. (1996), The Information Age: Economy, Society and culturere. I/ The rise of the Information society, Edizione Italiana Paperback (2008), UBE Paperback, Milano.

CASTELLS M. (1997), The Information Age: Economy, Society and culture: II/ The Power of Identity, Edizione italiana Paperback (2003), UBE Paperback, Milano.

CRETTIEZ X. et MUCCHIELLI L. (dir.) (2010), Les violences politiques en Europe : Un état des lieux, La Découverte, Paris.

COURLET C. (1988), “La frontière : couture ou coupure ?”, Economie et Humanisme, 301.

COURLET C. (1996), “Globalisation et frontière”, Sciences de la société, 37, Presses Universitaires du Mirail, Toulouse.

DEGRYSE C. (2007), Dictionnaire de l'Union européenne, de Boeck, Bruxelles.

ESPACE TEMPS (2004), http://test.espacestemps.net/articles/la-frontiere-un-objet-spatial-enmutation/

FRIEDMAN T. (2006), The World is Flat - The Globalized Word in the Twenty-First Century, Penguin Books, UK.

GUICHONNET P. et RAFFESTIN C. (1974), Géographie des frontières, PUF, Paris.

KEMP P. (2009), “The Journey to (No-) where. Constructing Place in a Space of Placelessness", Journal of New Frontiers in Spatial Concepts, 1, Universitätsverlag Karlsruhe.

LEIMGRUBER W. (2004), Between Global and Local - Marginality and Marginal Regions in the Context of Globalisation and Deregulation, Ashgate, Aldershot. 
NUGENT P. (2008), Smugglers, Secessionists and Loyal Citizens of the Ghana-Togo Frontier : the Lie of the borderlands since 1914, James Currey, Ohio University Press.

PFLIEGER G., CHENAL J., ATTARONI L., in http://www.espacestemps.net/en/articles/les-lieux-audetour-des-reseaux-en/ (31.5.13).

POPESCU G. (2012), Bordering and Ordening the Twenty-First Century - Understanding Borders, Rowman \& Littlefield Publishers, Lanham, Maryland.

PRESCOTT J.R.V. (1978), Boundaries and frontiers, London, Allen and Unwin.

RATTI R. (1991), Théorie du développement des régions-frontières, Centre de recherches en économie de l'espace de l'Université de Fribourg (Suisse).

RATTI R. and REICHMAN S. (eds.) (1993), Theory and Practice of Transborder Cooperation, Helbing \& Lichtenhahn, Basel und Frankfurt am Main.

RATTI R. (1994), "Spatial Effects of Frontiers : overview of different approaches and theories of border region development", in NIJKAMP P. (ed.), New Borders and Old Barriers in Spatial Development, Avebury, Aldershot.

RATTI R. (2003), "Does transfrontier cooperation exists in the electronic media field ? Theoretical reminders and socio-cultural gaps, in ISIG", Quartely of International Sociology, 3-4 Dic. 2003, Gorizia (Italy).

RETAILLÉ D. (2011), “Les transformations des formes de la limite”, Journal of Urban Studies, 6.

RETAILLÉ D. (2012), Les Lieux de la mondialisation, Le Cavalier Bleu, Paris.

DE ROUGEMONT D. (1971) “L’Europe est d'abord une unité de culture”, Cadmos, 43 (1988).

SASSEN S. (2006), Territory, Authority, Rights. From Medieval to Global Assemblages, Princeton University Press.

SASSEN S. (2010), "The Specialised Différences of Global Cities”, in MAGATTI M. and GHERARDI L., The City of Flows - Territories, Agencies and Institutions, Bruno Mondadori, Milano.

SENN L. (1990), in BRAMANTI A. et RATTI R., Verso un'Europa delle Regioni - La cooperazione economica transfrontaliera come opportunità e sfida, Franco Angeli, Milano.

SCHULER M., COMPAGNON A. et JEMELIN C. (1999), Les grandes régions de la Suisse, La Suisse dans le système des régions NUTS, OFS, Neuchâtel.

SIVEIRA M.L., (2010), “Globalisation, spécialisations territoriales productives et diversité”, in CRY P. et JOYAL A., Penser les territoires - En hommage à Georges Benko, Presses Universitaires du Québec, Québec.

TURNER F.J., (1921), The Frontier in American History, http://answers.yahoo.com/question/index? qid=20090129134528AA5ircn.

WORLD DEVELOPMENT REPORT (2009), Reshaping Economic Geography, The World Bank, Washington, DC.

\section{NOTES}

1. La réalité montre bien aussi des cas de survivance de territoires féodaux: (i) Etats nains (Monaco, Liechtenstein, San Marino, Andorre, Vatican) ; (ii) Statuts et territoriales spéciaux : la plupart supprimés (Hohenzollern, Avignon); (iii) Découpages "illogiques" (comme entre les 
cantons suisses de Fribourg et Vaud, une survivance territoriale de trois jours de conquête en 1536) ; (iv) Enclaves nationales (protégées par des frontières nationales). Exemple : la frontière nationale entre les Pays Bas et la Belgique montre une configuration très particulière dans le village de Baarle. Mais, en général, 200 ans de réformes territoriales ont modifié les anciennes structures.

2. Comme relevé par un de nos referees, des travaux récents montrent aussi des cas où les autorités locales ont joué un rôle le long des frontières, par exemple, entre la Gambie et le Sénégal ou le Ghana et le Togo (Nugent P., 2008).

3. Une première vérification est en cours pour la frontière suisse-italienne, par Alberto Bramanti, Università Bocconi, Milano et Remigio Ratti, Università della Svizzera Italiana, Lugano.

4. Les processus de territorialisation $\mathrm{du} 14^{\mathrm{e}}$ au $18^{\mathrm{e}}$ siècle ont vu la tendance à la création de territoires contigus, selon une trajectoire de longue durée et décalée entre les différentes régions européennes: division du travail (les villes); amélioration des routes (et de la navigation); évolution de la technologie militaire; déclin du pouvoir de l'Eglise ; changements d'échelle vers des pouvoirs plus grands.

\section{RÉSUMÉS}

Cet essai est une contribution à la théorisation des effets frontières, proposant une vision synthétique sous forme de typologie. Celle-ci thématise le changement de nature du rôle des frontières et de leur impact spatial: à partir des territoires contigus des frontières traditionnelles, on passe aux espaces fonctionnels de relations économiques et politiques désormais insérées dans la globalisation.

Après l'illustration de la construction du tableau typologique et des concepts qui sont à sa base, chaque cas est brièvement présenté dans ses caractéristiques concernant le rôle de la frontière, ses effets et l'éventuelle gestion des discontinuités.

This essay is a contribution to the theory of border effects, offering a synthetic vision in the form of a typology. It thematizes the changing nature of the role of borders and their spatial impact: from contiguous territories of traditional boundaries, moving to the functional areas of economic and political relationships now inserted into globalization.

Following the illustration of the construction of the typological table and the concepts that are at the base, each case is briefly presented in there characteristics on the role of the border, its effects and possible governance of discontinuities.

\section{INDEX}

Mots-clés : frontières, espace, territoires, Etat-nation, globalisation, sites, lieux, places

Keywords : border, space, territories, national-state, globalization, sites, lieux, places 


\section{AUTEURS}

\section{REMIGIO RATTI}

Professeur titulaire, Université de Fribourg et Università della Svizzera italiana, Lugano, remigio.ratti@unifr.ch

\section{MARTIN SCHULER}

Professeur émérite, Ecole Polytechnique Fédérale de Lausanne, martin.schuler@epfl.ch 